\title{
Backbone NMR assignments of HypF-N under conditions generating toxic and non-toxic oligomers
}

\author{
Jayneil R. Patel ${ }^{1} \cdot$ Yingqi Xu $^{1} \cdot$ Claudia Capitini $^{2} \cdot$ Fabrizio Chiti $^{2} \cdot$ Alfonso De Simone $^{1}$
}

Received: 5 October 2017 / Accepted: 24 April 2018 / Published online: 21 May 2018

(c) The Author(s) 2018

\begin{abstract}
The HypF protein is involved in the maturation and regulation of hydrogenases. The N-terminal domain of HypF (HypF$\mathrm{N}$ ) has served as a key model system to study the pathways of protein amyloid formation and the nature of the toxicity of pre-fibrilar protein oligomers. This domain can aggregate into two forms of oligomers having significantly different toxic effects when added to neuronal cultures. Here, NMR assignments of HypF-N backbone resonances are presented in its native state and under the conditions favouring the formation of toxic and non-toxic oligomers. The analyses of chemical shifts provide insights into the protein conformational state and the possible pathways leading to the formation of different types of oligomers.
\end{abstract}

Keywords Biomolecular NMR $\cdot$ Protein assignment $\cdot$ HypF-N $\cdot$ Amyloids $\cdot$ Protein oligomers

\section{Introduction}

HypF plays a chaperone role in the biogenesis of nickel-iron $[\mathrm{NiFe}]$ insertion in hydrogenase enzymes from $E$. coli (Maier and Böck 1996). The structure of HypF, lacking its $\mathrm{N}$-terminal acylphosphatase domain, has been previously elucidated by X-ray crystallography (Petkun et al. 2011) (PDB code: 3TSP, 3TSQ, 3TSU, 3TTD, 3TTF). The $\mathrm{N}$-terminal domain of $\mathrm{HypF}(\mathrm{HypF}-\mathrm{N})$ is a small $11 \mathrm{kDa} \alpha / \beta$ protein of 91 residues (Rosano et al. 2002) that is structured in a $\beta$-sheet of five strands (S1-S5) and $2 \alpha$-helices (H1 and $\mathrm{H} 2$ ) in a $\beta \alpha \beta \beta \alpha \beta$ topology (PDB code: 1GXT, 1GXU) (Rosano et al. 2002). While HypF-N is not associated to any disease, it has been extensively used to elucidate the underlying biomolecular processes of neurodegenerative disorders (Chiti et al. 2001; Campioni et al. 2010; Zampagni et al. 2011). It is indeed well established that the aggregation of otherwise soluble proteins into amyloid fibrils is associated with a number of neurodegenerative conditions,

Alfonso De Simone

adesimon@imperial.ac.uk

1 Department of Life Sciences, Imperial College London, South Kensington, London SW72AZ, UK

2 Section of Biochemistry, Department of Experimental and Clinical Biomedical Sciences, University of Florence, Viale Morgagni 50, 50134 Firenze, Italy including Alzheimer's and Parkinson's diseases, and nonneuropathic conditions such as diabetes type II (Chiti and Dobson 2017). It is now generally acknowledged that the most pernicious species along the pathways of amyloid formation are the small diffusible pre-fibrilar oligomers (Campioni et al. 2010; Fusco et al. 2017). In this context, HypF-N has unique features to enable the elucidation of the molecular basis of the toxicity of protein oligomers, as it can form two different types of protein oligomers that, while being similar in composition, shape, size and morphology, have significantly different toxic effects when incubated with cellular cultures, with only one showing toxic effects. Such a biological diversity has been attributed to the selective interaction with the cellular membrane, with only the toxic oligomers being able to penetrate the membrane and cause an influx of $\mathrm{Ca}^{2+}$ ions (Cecchi et al. 2005; Canale et al. 2006; Campioni et al. 2010; Zampagni et al. 2011). Despite the relevance of this protein in the context of amyloid diseases, the assignments of the NMR resonances of HypF-N under the conditions $\mathrm{A}$ and $\mathrm{B}$, which respectively generate toxic and non-toxic oligomers, are not available from the BMRB. In the present work we present a detailed characterisation of the general structural properties of HypF-N in its native state and under the two oligomerising conditions. The analysis of chemical shifts clearly identifies a substantial degree of structural and dynamical differences between the protein monomers investigated under the conditions leading to toxic 
and non-toxic oligomers. This work will boost further highresolution characterisations in these two states.

\section{Methods and materials}

${ }^{1} \mathrm{H},{ }^{13} \mathrm{C},{ }^{15} \mathrm{~N}$, isotopically labelled HypF-N was expressed and purified as previously reported (Calloni et al. 2008), using an N-terminal His-tag construct that was expressed in the $E$. coli strain M15[PREP4] (Qiagen), grown in M9 minimal media by using ${ }^{15} \mathrm{~N}$-enriched ammonium chloride and ${ }^{13} \mathrm{C}$-enriched glucose, and purified using a nickel column (Sigma Aldrich). The HypF-N protein was then cleaved from the nickel resin with thrombin from bovine plasma (Sigma Aldrich) overnight at $4{ }^{\circ} \mathrm{C}$ in phosphate buffer. Thrombin cleavage generates a non-native $\mathrm{N}$-terminal sequence (GlySer-Ala instead of Met-Ala). This construct is equivalent to that employed in the study identifying the conditions (A and $\mathrm{B}$ ) to generate toxic and non-toxic HypF-N oligomers (Campioni et al. 2010). The eluted HypF-N was then buffer exchanged into $5 \mathrm{mM}$ sodium acetate, $2 \mathrm{mM}$ dithiothreitol (DTT) buffer at pH 5.5.

NMR measurements were performed on ${ }^{13} \mathrm{C},{ }^{15} \mathrm{~N}$ labelled HypF-N samples at a concentration of $200 \mu \mathrm{M}$. Three independent assignments of the backbone resonances were performed, including native $(5 \mathrm{mM}$ sodium acetate, $2 \mathrm{mM}$ DTT, $50 \mathrm{mM}$ sodium phosphate $\mathrm{pH}$ 5.5), toxic condition a
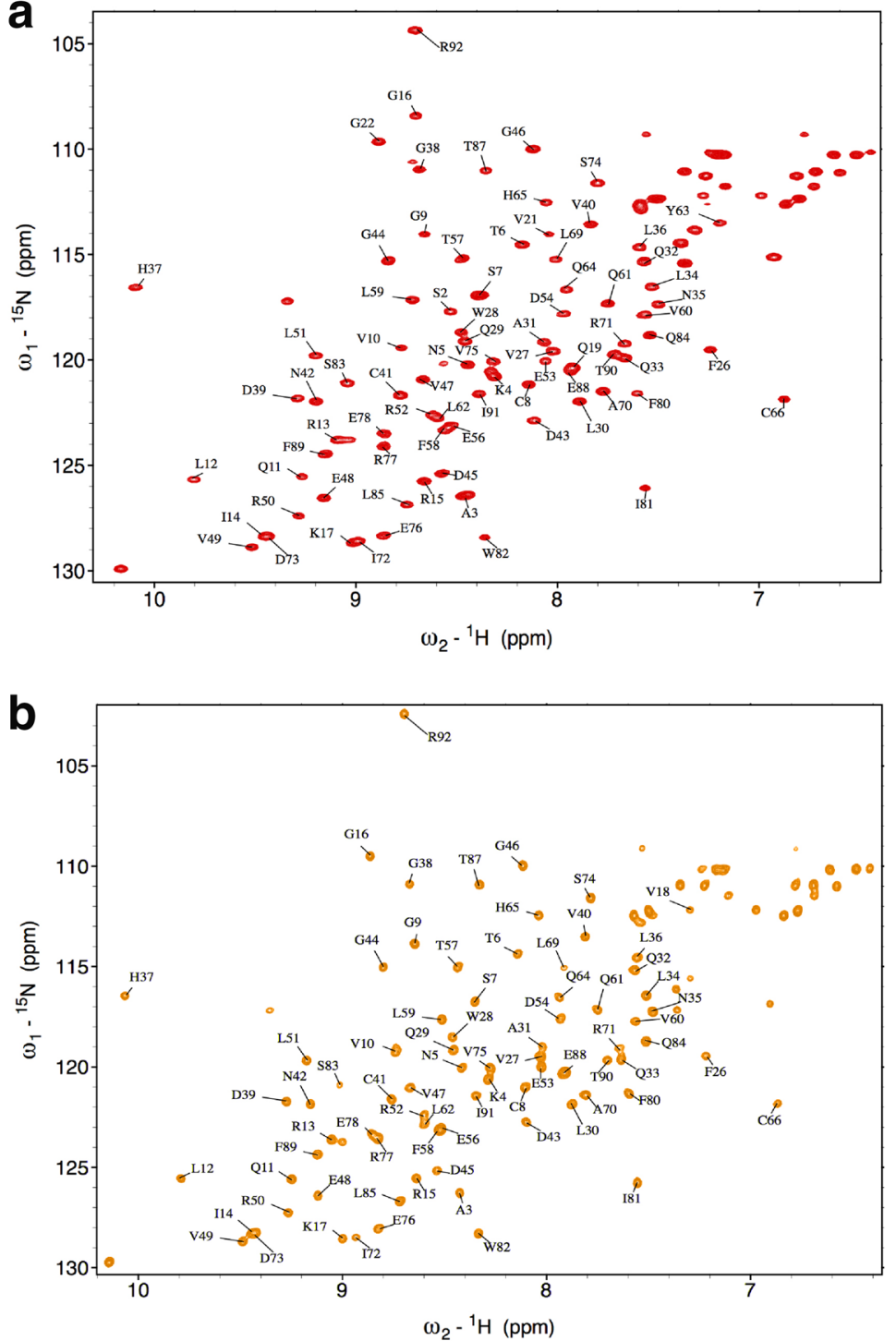

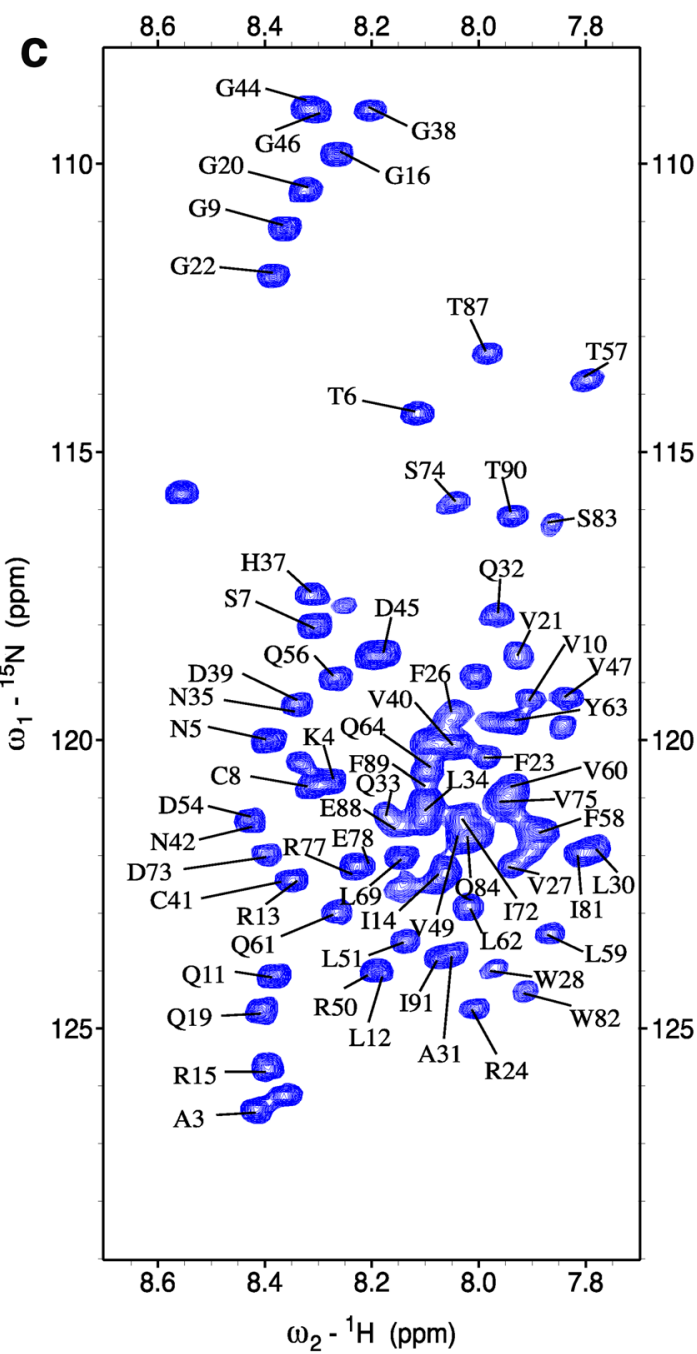

Fig. 1 Assigned 2D ${ }^{1} \mathrm{H}-{ }^{15} \mathrm{~N}$ HSQC spectra of HypF-N recorded at $25^{\circ} \mathrm{C}$ at the ${ }^{1} \mathrm{H}$ frequency of $600 \mathrm{MHz}$. a Native state. b Condition A. c Condition $\mathrm{B}$ 
A (50 mM sodium acetate, $2 \mathrm{mM}$ DTT, 12\% 2,2,2-trifluoroethanol (TFE) (v/v), pH 5.5) and non-toxic condition B [330 mM sodium chloride, $20 \mathrm{mM}$ trifluoroacetic acid (TFA), $\mathrm{pH}$ 1.7]. Each sample contained $10 \%(\mathrm{v} / \mathrm{v}) \mathrm{D}_{2} \mathrm{O}$ for the NMR lock. For the aggregation prone conditions $\mathrm{A}$ and $\mathrm{B}$, NMR measurements were performed in a time frame corresponding to the lag phase for aggregation, which enables minimal monomer depletion from the solution. Under the conditions employed in this work, the lag phases were $\sim 16$ and $\sim 24 \mathrm{~h}$ for condition A and B, respectively. Measurements of 3D NMR spectra were therefore set to be carried out within these time ranges, requiring fresh samples to be prepared for each 3D measurement.

Assignment of the backbone resonances under these three conditions was performed by a combination of ${ }^{1} \mathrm{H}-{ }^{15} \mathrm{~N}$ HSQC, CBCA(CO)NH, HNCACB, HNCO, HN(CA)CO and HNHA spectra, collectively providing chemical shifts for ${ }^{1} \mathrm{H} \alpha,{ }^{13} \mathrm{C} \alpha,{ }^{13} \mathrm{C} \beta,{ }^{1} \mathrm{HN},{ }^{13} \mathrm{CO}$ and ${ }^{15} \mathrm{~N}$ atoms. For the nontoxic condition $\mathrm{B}$, an additional HNcocaNH spectrum (Sun et al. 2005) was recorded to aid sequential assignment. NMR was performed at $25{ }^{\circ} \mathrm{C}$ using Bruker AVANCE spectrometers operating at proton frequencies of 600 or $800 \mathrm{MHz}$,

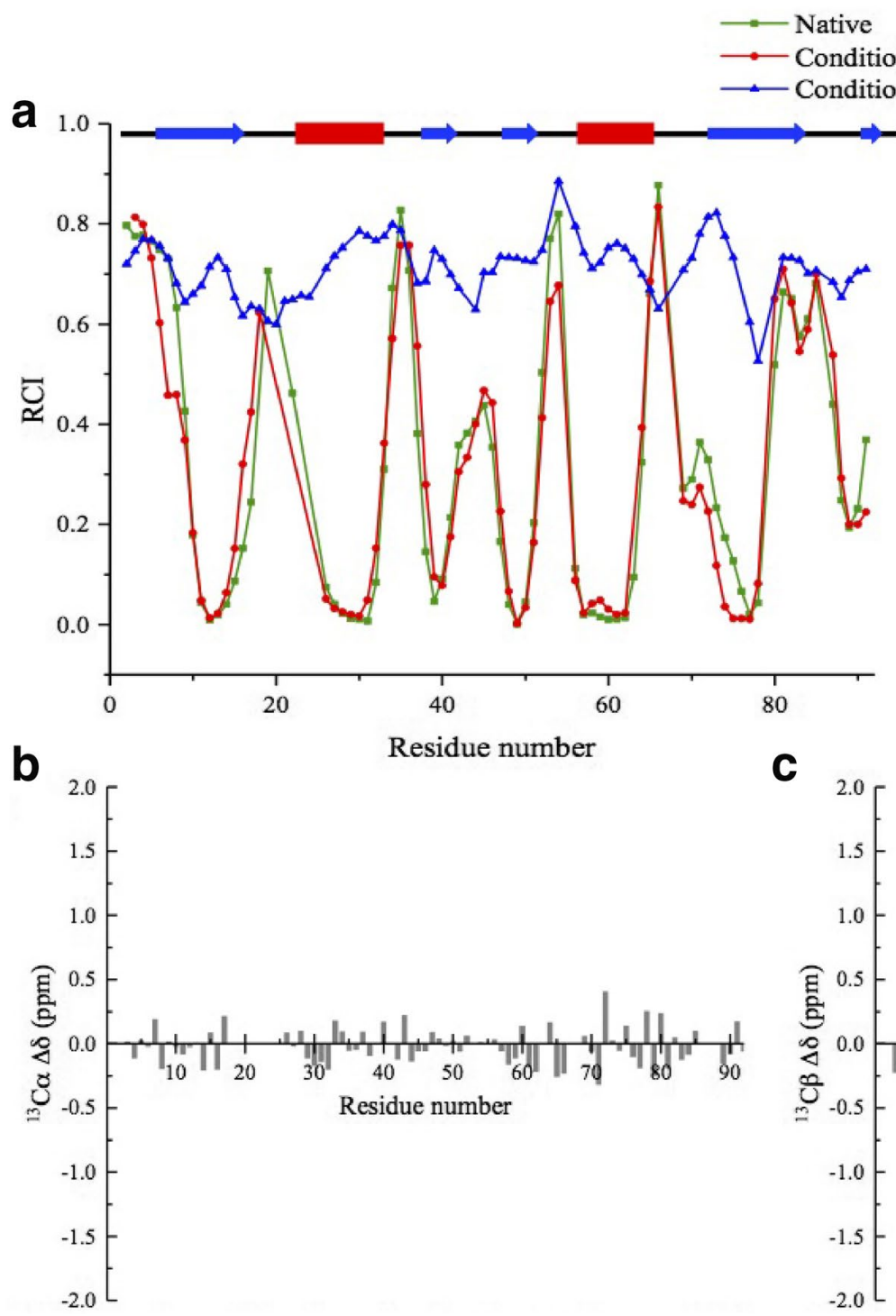

- Native

$\longrightarrow$ Condition A

Condition B

b

Fig. 2 a Random coil index estimated using the population of coil regions from $\delta 2 \mathrm{D}$ (Camilloni et al. 2012). Native state and aggregation prone states of HypF-N (condition A and condition B) are shown with green, red and blue lines, respectively. Lines are used to connect the data for which RCI values have been calculated based on chemical shifts. The schematic of the native protein secondary structure is

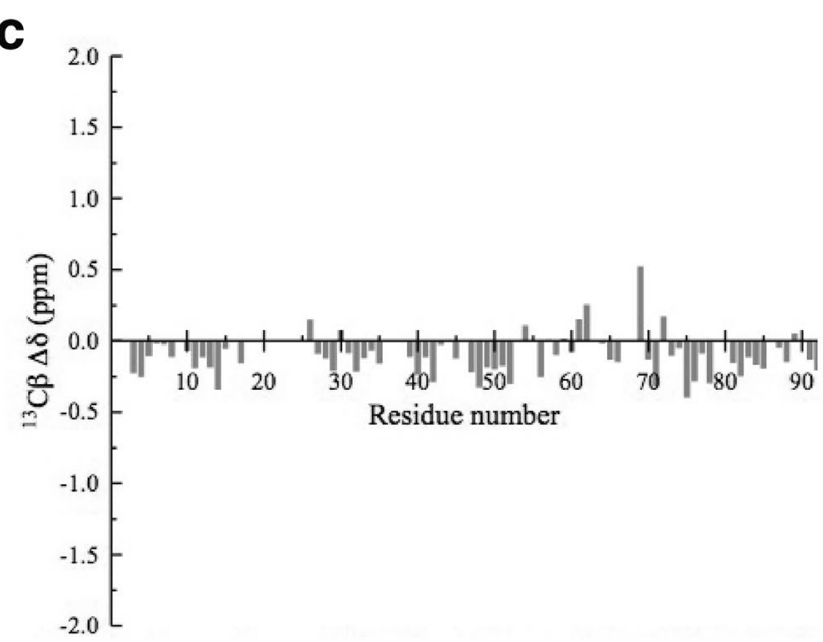

shown illustrating the $\alpha$-helical segments (red blocks) and $\beta$-sheet strands (blue arrows). b-c) Differences between the ${ }^{13} \mathrm{C} \alpha$ (b) and ${ }^{13} \mathrm{C} \beta$ (c) chemical shifts measured for the native state and condition A of HypF-N. Data has been excluded where assignments are missing in one or both conditions 
Table 1 Number of assigned nuclei

\begin{tabular}{lllllll}
\hline & ${ }^{1} \mathrm{H} \alpha$ & ${ }^{13} \mathrm{C} \alpha$ & ${ }^{13} \mathrm{C} \beta$ & ${ }^{13} \mathrm{CO}$ & ${ }^{15} \mathrm{~N}$ & ${ }^{1} \mathrm{HN}$ \\
\hline Native $^{\mathrm{a}}$ & 79 & 78 & 81 & 79 & 81 & 81 \\
Condition A & 72 & 77 & 78 & 77 & 77 & 77 \\
Condition B & 62 & 78 & 79 & 78 & 78 & 78 \\
\hline
\end{tabular}

${ }^{a}$ Unassigned or missing backbone amide resonances in the ${ }^{1} \mathrm{H}-{ }^{15} \mathrm{~N}$ HSQC: Native: 1, 18, 20, 23, 24. Condition A: 1, 2, 19-24, 63. Condition B: 1, 29, 36, 43, 53 76, 80, 92

both equipped with a triple resonance cryoprobe. NMR data were processed using NMRPipe (Delaglio et al. 1995) and analysed using CCPNAnalysis (Vranken et al. 2005).

\section{Results}

Under native conditions, 81 (out of 86 non-proline) ${ }^{1} \mathrm{H}-{ }^{15} \mathrm{~N}$ correlations were assigned across the protein sequence (Fig. 1a). The peak dispersion in the ${ }^{1} \mathrm{H}-{ }^{15} \mathrm{~N}-\mathrm{HSQC}$ spectrum indicates that HypF-N is well-folded in its native state, as also confirmed by the RCI analysis made using $82 \mathrm{D}$ (Camilloni et al. 2012) (Fig. 2a). Under condition A, the spectral properties suggest that HypF-N is structured in a native-like conformational state (Fig. 1a-b), with ${ }^{1} \mathrm{H}^{15} \mathrm{~N}$ resonances close to those of the native state, except for the catalytic phosphate binding site (residues 18-24), for which no NMR resonances are detected under these conditions. Indeed, the lack of phosphate ions in the condition A likely generates a local conformational exchange that enhances the relaxation of the resonances of the loop 18-24 leading to significant peak broadening, observed in homologous acylphosphotase enzymes (Fusco et al. 2012; De Simone et al. 2011). The similarity of spectral properties between native state and condition $\mathrm{A}$ is also shown by the difference in chemical shifts for ${ }^{13} \mathrm{C} \alpha$ and ${ }^{13} \mathrm{C} \beta$ (Fig. 2b, c). In contrast, under condition B, HypF-N shows spectral properties that are indicative of an unfolded protein (Fig. 1c), as previously observed (Calloni et al. 2008). The assignment statistics for conditions A and B are listed in Table 1.

Using the chemical shift values, random coil index was calculated as the population of coil regions in $82 \mathrm{D}$ (Camilloni et al. 2012) (Fig. 2a). This analysis indicates that, in contrast to the native state and condition A, showing structured and coil regions that are consistent with those identified in the X-ray structure of HypF-N (PDB code: 1GXT; Rosano et al. 2002), condition B exhibits significantly high values of RCI throughout the sequence, which is indicative of a highly flexible and disordered state.

In summary, NMR assignments of HypF-N resonances under different oligomerising conditions and in the native state have provided crucial insights into the conformational and structural properties of the protein in its physiological and aggregation-prone states. The assignments will enable further investigations to reveal the detailed mechanisms leading to the formation of toxic and nontoxic HypF-N oligomers.

The assignments have been deposited to the BMRB under the accession codes: 27139 (native), 27137 (toxic) and 27138 (non-toxic).

Acknowledgements We acknowledge support from the Leverhulme Trust (RPG-2015-350) and the UK Biotechnology and Biological Sciences Research Council (BB/M023923/1) and Wellcome Trust (104933/Z/14/Z).

Open Access This article is distributed under the terms of the Creative Commons Attribution 4.0 International License (http://creativeco mmons.org/licenses/by/4.0/), which permits unrestricted use, distribution, and reproduction in any medium, provided you give appropriate credit to the original author(s) and the source, provide a link to the Creative Commons license, and indicate if changes were made.

\section{References}

Calloni G, Lendel C, Campioni S, Giannini S, Gliozzi A, Relini A, Vendruscolo M, Dobson CM, Salvatella X, Chiti F (2008) Structure and dynamics of a partially folded protein are decoupled from its mechanism of aggregation. J Am Chem Soc 130:13040-13050

Camilloni C, De Simone A, Vranken WF, Vendruscolo M (2012) Determination of secondary structure populations in disordered states of proteins using nuclear magnetic resonance chemical shifts. Biochemistry 51:2224-2231

Campioni S, Mannini B, Zampagni M, Penselfini A, Parrini C, Evangelisti E, Relini A, Stefani M, Dobson CM, Cecchi C, Chiti F (2010) A causative link between the structure of aberrant protein oligomers and their toxicity. Nat Chem Biol 6:140-147

Canale C, Torrassa S, Rispoli P, Relini A, Rolandi R, Bucciantini M, Stefani M, Gliozzi A (2006) Natively folded HypF-N and its early amyloid aggregates interact with phospholipid monolayers and destabilize supported phospholipid bilayers. Biophys $\mathbf{J}$ 91(12):4575-4588

Cecchi C, Baglioni S, Fiorillo C, Pensalfini A, Liguri G, Nosi D, Rigacci S, Bucciantini M, Stefani M (2005) Insights into the molecular basis of the differing susceptibility of varying cell types to the toxicity of amyloid aggregates. J Cell Sci 118:3459-3470

Chiti F, Dobson CM (2017) Protein misfolding, Amyloid Formation and Human Disease: A Summary of Progress Over the Last Decade. Annu Rev Biochem 86:27-68

Chiti F, Bucciantini M, Capanni C, Taddei N, Dobson CM, Stefani M (2001) Solution conditions can promote formations of either amyloid protofilaments or mature fibrils from the HypF-N terminal domain. Protein Sci 10:2541-2547

De Simone A, Dhulesia A, Soldi G, Vendruscolo M, Hsu ST, Chiti F, Dobson CM (2011). Experimental free energy surfaces reveal the 
mechanisms of maintenance of protein solubility. Proc Natl Acad Sci USA 108(52):21057-21062

Delaglio F, Grzesiek S, Vuister GW, Zhu G, Pfeifer J, Bax A (1995) NMRPipe: a multidimensional spectral processing system based on UNIX pipes. J Biomol NMR 6(3):277-293

Fusco G, De Simone A, Hsu ST, Bemporad F, Vendruscolo M, Chiti F, Dobson CM (2012) ${ }^{1} \mathrm{H},{ }^{13} \mathrm{C}$ and ${ }^{15} \mathrm{~N}$ resonance assignments of human muscle acylphosphatase. Biomol. NMR Assign 6(1):27-29

Fusco G, Chen SW, Williamson PT, Cascella R, Perni M, Jarvis JA, Cecchi C, Vendruscolo M, Chiti F, Cremades N, Ying L, Dobson CM, De Simone A (2017) Structural basis of the membrane disruption and cellular toxicity by $\alpha$-synuclein oligomners. Science 358(6369):1440-1443

Maier T, Böck A (1996) Nickel incorporation into hydrogenases. In: Hausinger RP, Eichhorn GL, Marzilli LG (eds) Advances in inorganic biochemistry: mechanisms of metallocenter assembly. VCH Publishers Inc., New York, pp 173-192

Petkun S, Shi R, Li Y, Asinas A, Munger C, Zhang L, Waclawek M, Soboh B, Sawers RG, Cygler M (2011) Structure of hydrogenase maturation protein HypF with reaction intermediates shows two active sites. Structure 19:1773-1783

Rosano C, Zuccotti S, Bucciantini M, Stefani M, Rampioni G, Bolognesi M (2002) Crystal structure and anion binding in the prokaryotic hydrogenase maturation factor HypF acylphosphatase-like domain. J Mol Biol 321:785-796

Sun ZY, Frueh DP, Selenko P, Hoch JC, Wagner G (2005) Fast assignment of ${ }^{15} \mathrm{~N}$-HSQC peaks using high-resolution 3D HNcocaNH experiments with non-uniform sampling. J Biomol NMR 33:43-50

Vranken WF, Boucher W, Stevens TJ, Fogh RH, Pajon A, Llinas M, Ulrich EL, Markley JL, Ionides J, Laue ED (2005) The CCPN data model for NMR spectroscopy: development of a software pipeline. Proteins 59:687-696

Zampagni M, Cascella R, Casamenti F, Grossi C, Evangelisti E, Wright D, Becatti M, Liguri G, Mannini B, Campioni S, Chiti F, Cecchi C (2011) A comparison of the biochemical modifications caused by toxic and non-toxic protein oligomers in cells. J Cell Mol Med 15(10):2106-2116 